\title{
Improvement of Several Classical Matching Algorithms for Underground Positioning
}

\author{
Jie Huang ${ }^{1, *}$, Ziyuan Tong ${ }^{2}$, Shoufeng Tang ${ }^{1}$, Qingyong Liu ${ }^{1}$, Minming Tong ${ }^{1}$ and Mingyu Ji ${ }^{3}$ \\ ${ }^{1}$ School of Information and Control Engineering China University of Mining and Technology, Xuzhou, Jiangsu Province, 221008, \\ China \\ ${ }^{2}$ School of Electrical Engineering and Telecommunications, The University of New South Wales, NSW2052, Australia \\ ${ }^{3}$ Xuzhou Hanlin Technology CO., LTD. \\ ${ }^{*}$ Corresponding author
}

\begin{abstract}
It is an urgent task for the coal industry in China and even the world to realize the position of the injured and the excavation equipment under the mine. Due to its advantages of high efficiency, low cost, and suitable accuracy, binocular stereo vision measurement methods have become a popular trend in recent years to achieve dynamic target positioning. The stereo matching problem is a key problem in the binocular stereo vision system. This paper analyzes and improves several classical matching algorithms, and combines the improved algorithms together for positioning research. Finally through simulation experiments, an area adaptive matching algorithm with advantages is obtained.
\end{abstract}

Keywords—mine; binocular stereo vision; matching algorithm; position

\section{INTRODUCTION}

According to reports, on April 10, 2018, in the afternoon, a safety accident occurred in the Qinglinwan coal mine in Pingbaying Township, Xianfeng County. Four people were trapped underground. Due to the lack of timely access to the specific location of the trapped persons, the search and rescue work was slow. By the time the search and rescue work was completed, all four had no vital signs. It can be seen that the positioning technology under the mine is the guarantee for the safe mining of mines. As a major country for coal mining and use, the development of underground positioning technology will determine the future coal mining safety rate in China ${ }^{[1]}$.Binocular stereo vision is one of the key technologies of machine vision. It performs a reconstruction of the threedimensional scene with the obtained two-dimensional image ${ }^{[2]}$.This technology can avoid the direct participation of people in dangerous environments, so its application in aerospace mapping, military applications, medical imaging and industrial inspection is also increasingly widespread ${ }^{[3]}$.

\section{AdAPtiVe Weight Stereo Matching Algorithm}

\section{A. Adaptive Weight Method}

The adaptive weighting method ${ }^{[4]}$ is the so-called principle of weight distribution. The idea is to use weight and distance information to weight each pixel. The weight of the domain pixel ${ }^{[5]}$ is defined as follows:

$$
W(p, q)=f\left(\Delta c_{p q}, \Delta g_{p q}\right)=f_{s}\left(\Delta c_{p q}\right) \bullet f_{p}\left(\Delta g_{p q}\right)
$$

Where $\Delta \mathrm{c}_{p q}$ denotes the color difference between the point $\mathrm{p}$ to be matched and the pixel point $\mathrm{q}$ in the matching window, $\Delta \mathrm{g}_{p q}$ denotes the Euclidean distance between the two points, $f_{s}\left(\Delta c_{p q}\right)$ is the model established for the color similarity of the pixels $\mathrm{p}$ and $\mathrm{q}$, and $f_{\mathrm{p}}\left(\Delta g_{p q}\right)$ is A model for establishing the Euclidean distance of pixel points $\mathrm{p}$ and $\mathrm{q}^{[6]}$.

$$
f_{s}\left(\Delta c_{p q}\right)=\exp \left(-\frac{\Delta c_{p q}}{\gamma_{c}}\right) f_{p}\left(\Delta g_{p q}\right)=\exp \left(-\frac{\Delta g_{p q}}{\gamma_{p}}\right)
$$

Where $\Delta \mathrm{c}_{p q}$ denotes the similarity of the two pixel points $\mathrm{c}_{p}=\left\lfloor L_{p}, a_{p}, b_{p}\right\rfloor$ and $c_{q}=\left\lfloor L_{q}, a_{q}, b_{q}\right\rfloor$ in the CIELab color, $\Delta g_{p q}$ denotes the Euclidean distance of the pixel points p and q, and $\gamma_{\mathrm{c}}$ and $\gamma_{\mathrm{p}}$ denote the similarity threshold and the affinity threshold between the two pixel points, respectively. Finally get the weight $w(p, q)^{[7]}$.

$$
w(p, q)=\exp \left(-\left(\frac{\Delta c_{p q}}{\gamma_{c}}+\frac{\Delta g_{p q}}{\gamma_{p}}\right)\right)
$$

The essence of ASW's aggregation strategy is clusters pixels with similar color or distance to match point together. As shown in Figure I.
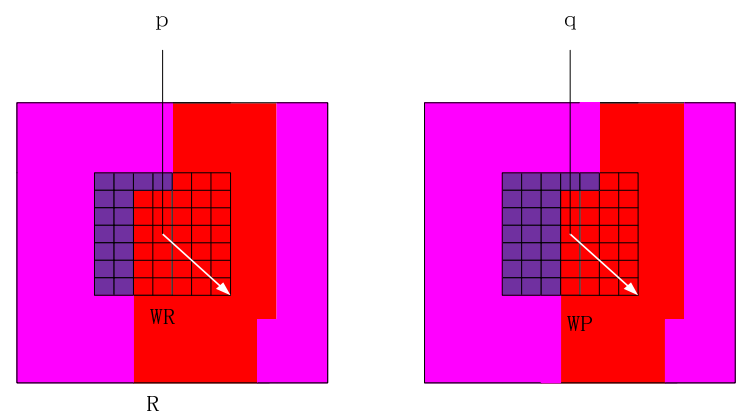

FIGURE I. ASW AGGREGATION STRATEGY 
In order to improve the accuracy, all the weight values of the left and right graph matching windows are taken into account when calculating the matching $\operatorname{cost}^{[8]}$.

$$
E\left(p, p_{d}\right)=\frac{\sum_{q \in N_{p}, \bar{q}_{d} \in N_{p d}} \mathfrak{W}(p, q) \mathfrak{W}\left(p_{d}, q_{d}\right) e\left(p, p_{d}\right)}{\sum_{q \in N_{p}, \bar{q}_{d} \in N_{p d}} \operatorname{W}(p, q) \mathfrak{W}\left(p_{d}, q_{d}\right)} \quad e\left(p, p_{d}\right)=\min \left\{\sum_{c \in\{r, q, b\}}\left|I_{c}(p)-I_{c}\left(p_{d}\right), T\right|\right\}
$$

As shown in Figure I, $p_{d}$ and $\mathrm{q}_{d}$ in the right figure are pixel points corresponding to $\mathrm{p}$ and $\mathrm{q}$ in the left figure when the disparity is $\mathrm{d}, \mathrm{e}\left(p, p_{d}\right)$ indicates a matching cost between single points, and color truncation is performed by cutting off the absolute error. ASW matching results shown in Figure II.
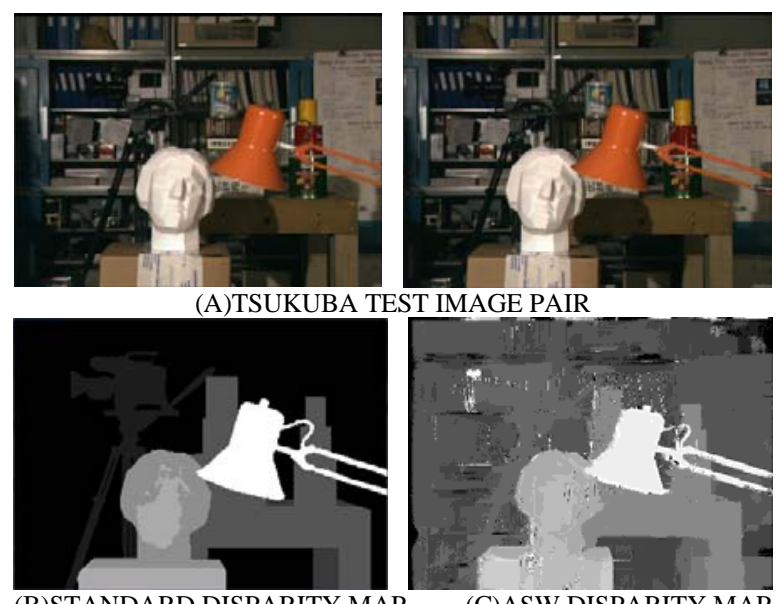

(B)STANDARD DISPARITY MAP

(C)ASW DISPARITY MAP

FIGURE II. ASW MATCH RESULT GRAPH

\section{B. Improved Adaptive Weighting Algorithm}

The improved adaptive weighting algorithm proposes a virtual window technique to reduce the time complexity of ASW. Simultaneously, the adaptive weight is simplified to reduce the false matching rate ${ }^{[9]}$.

The core idea of the virtual window technique ${ }^{[10]}$ is to split the image with a pair of horizontal and vertical straight lines and divide it into a number of rectangular regions, each of which contains one or more pixels. As shown in Figure III.

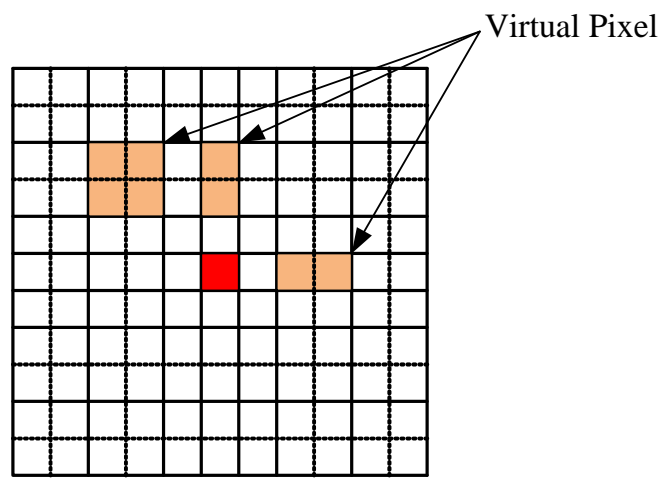

FIGURE III. VIRTUAL WINDOW
The color value of a pixel point $\mathrm{x}$ in the window $\mathrm{P}$ on the $\mathrm{c}$ channel can be expressed as follows:

$$
I_{c}^{(p)}(x, y)=\sum_{f_{x} \leq x^{\prime} \leq f_{(x+1)} f_{y} \leq y^{\prime} \leq f(y+1)} I_{c}\left(x_{0}+x^{\prime}, y_{0}+y^{\prime}\right) /\left(\delta_{x} \times \delta_{y}\right)
$$

Where $\delta_{\mathrm{x}}=f_{(x+1)}-f_{x}$ denotes the side length of the split window in the horizontal direction and $\delta_{y}=f_{(y+1)}-f_{y}$ denotes the side length of the split window in the vertical direction.

The improved ASW algorithm sets the weight of pixels connected to the pixel to be matched and the similarity of color information of pixels to 1 ; the weights of other pixels consider only the Euclidean distance without considering the color information, making the algorithm more robust. Set a color threshold $D_{0}$.

$$
\mathrm{d}(p, q)=\sqrt{\left(L_{p}-L_{q}\right)^{2}+\left(a_{p}-a_{q}\right)^{2}+\left(b_{p}-b_{q}\right)^{2}} \leq D_{0}
$$
that is:

If the matching center $\mathrm{p}$ is connected to the neighbor pixel q,

$$
L_{\mathrm{p}}(q)= \begin{cases}1 & d(p, q) \leq D_{0} \\ 0 & \text { 其他 }\end{cases}
$$

The set $\mathrm{S}$ is composed of all the pixels in the matching support window that are in communication with the pixels to be matched. The simplified adaptive weights are as follows:

$$
w(p, q)= \begin{cases}1 & q \in S \\ \exp \left(-\frac{\Delta g_{p q}}{\gamma_{p}}\right) & q \notin S\end{cases}
$$

The simplified self-adaptive weight makes the matching accuracy of the algorithm at the edge greatly improved, because in the supporting window of the point to be matched at the edge, as long as it is connected with the point to be matched, regardless of its distance to be matched, it The weight is always 1 , which solves the problem of having a small effect on the final matching result due to the low weight caused by the long distance of good point. At the same time, considering that the point to be matched is in a high-texture area, because the texture is dense, the number of pixels connected to the pixel to be matched decreases, although the pixel to be matched is not connected to many surrounding pixels, but because the Euclidean distance is very close, it makes The weights still have a great effect on the matching results and reduce the false matching rate ${ }^{[11]}$.

It can be seen from Figure IV that the improved ASW algorithm greatly reduces the mis-match rate and noise sensitivity of the edge region and improves the matching accuracy at the same time. 


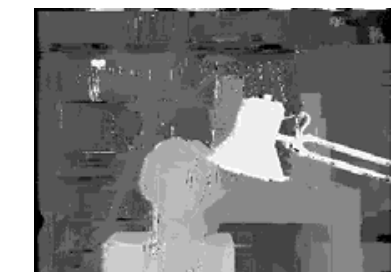

(A)ASW METHOD DISPARITY MAP

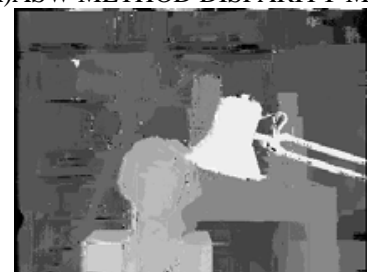

(B)IMPROVED ASW DISPARITY MAP

FIGURE IV. IMPROVED ASW MATCH RESULT MAP

\section{Segmentation-BASED NEAR REAL-TIME Stereo MATCHING ALGORITHM}

Some people have proposed near real-time stereo matching algorithm based on effective cost aggregation(ECA) ${ }^{[12]}$. This clustering method selects a variable support, which can be segmented from the image color cost information, and the color aggregation cost function is as follows:

$$
C_{s}(p, q, d)=\sum_{p_{i} \in S_{p}} \min \left(\delta\left(p_{i}, q_{i, d}\right), T_{r}\right)
$$

Where $S_{\mathrm{p}}$ is the block where pixel point $\mathrm{p}$ is to be matched, $\mathrm{d}$ is the disparity between pixel point $\mathrm{p}$ and pixel point $\mathrm{q}, \delta(p, q)$ is the distance between pixel point $\mathrm{p}$ and pixel point q, $T_{r}$ is the fixed threshold, and $T_{r}$ is set to enhance the robustness of the algorithm. The author uses the distance function of RGB space ${ }^{[13]}$ :

$$
\delta(p, q)=\left|R_{p}-R_{q}\right|+\left|G_{p}-G_{q}\right|+\left|B_{p}-B_{q}\right|
$$

As shown in Figure V, matching results using only the $\mathrm{s}$ function as the aggregation cost have poor matching performance in high texture areas.

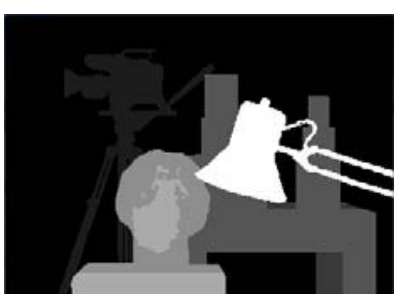

(A)STA

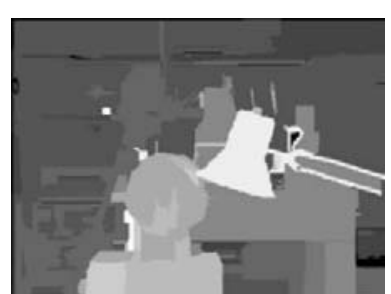

(B)ECA DISPARITY MAP
In order to reduce the mis-matching of the algorithm in high-texture areas, supplementary cost functions can be added.

$$
C_{w}(p, q, d)=\sum_{p_{i} \in W_{p}(r)} \min \left(\delta\left(p_{i}, q_{i, d}\right), T_{r}\right)
$$

The resulting ECA total matching cost function is as follows:

$$
C_{\text {aggr }}(p, q, d)=\frac{C_{s}(p, q, d)}{n\left(S_{p}\right)}+\alpha \bullet \frac{C_{w}(p, q, d)}{(2 r+1)^{2}}
$$

As shown in Figure VI, it can be seen that the mismatch of the ECA added to the compensation window to the high texture area is significantly reduced. At the same time, false matches at the edges increase ${ }^{[14]}$.
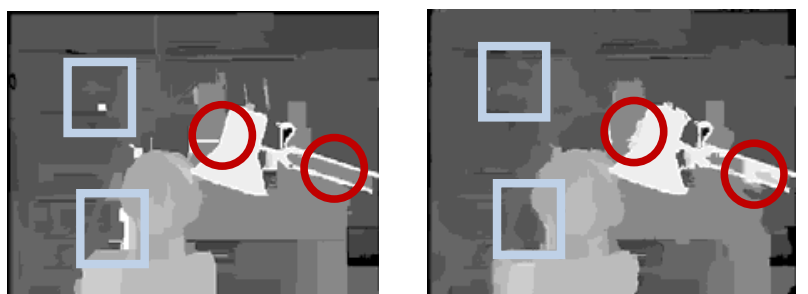

(A)UNCOMPENSATED WINDOW (B)ADD COMPENSATION WINDOW

FIGURE VI. ECA MATCHING RESULT GRAPH AFTER ADDING COMPENSATION WINDOW

\section{Regional AdAPtive Matching Method}

Considering that the matching accuracy of ECA in the edge region is low, it satisfies the real-time requirements of mine operations. The improved ASW algorithm is not high in time efficiency, but it has high accuracy and good matching effect at the edge. Therefore, the region adaptive matching method is proposed, and the improved ASW algorithm is used to perform stereo matching at the edge, and the ECA algorithm is used for stereo matching at the non-edge ${ }^{[15]}$.The combined effect chart obtained is shown in Figure VII.
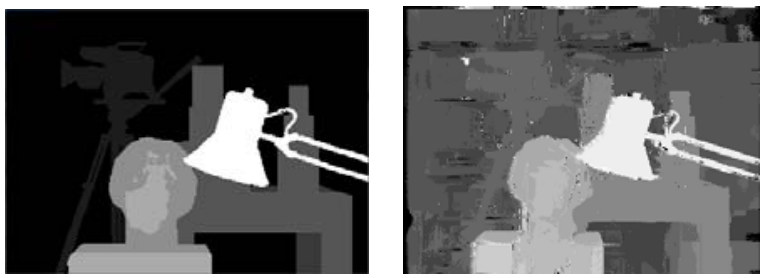

(A)STANDARD DISPARITY MAP(B)IMPROVED ASW DISPARITY MAP

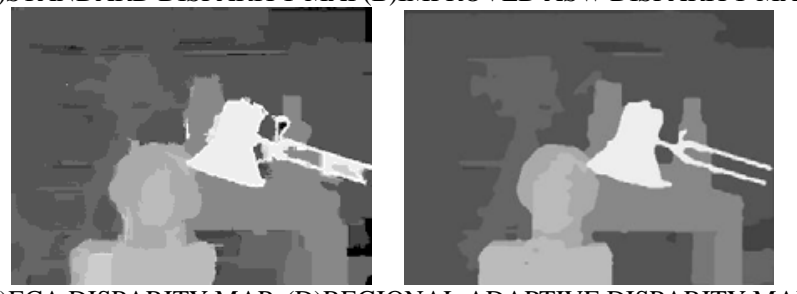

(C)ECA DISPARITY MAP (D)REGIONAL ADAPTIVE DISPARITY MAP

FIGURE VII. THE EFFECT OF DIFFERENT ALGORITHMS

It can be seen from Figure VII that the region self-adaption method fully utilizes the complementary advantages of the improved ASW algorithm and the ECA stereo matching algorithm. As can be seen from the matching result graph, this 
method achieves a good matching effect in the high texture area, the low texture area, and the edge area. At the same time, the method has a running time of about 4 seconds and is balanced in the matching accuracy and time efficiency.

\section{Simulation ExPERIMENT AND DATA ANALYSiS}

Due to the limitation of actual conditions, experiments cannot be conducted under the mine, so the hydraulic support is used as an excavation equipment under the mine for simulation experiments ${ }^{[16]}$.The excavator simulation scene image from the left and right cameras is shown in Figure VIII.

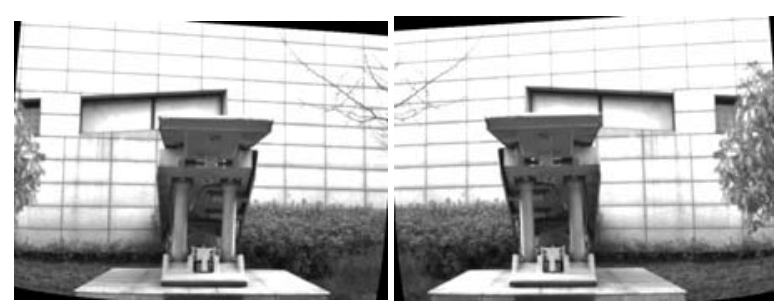

FIGURE VIII. THE CORRECTED LEFT AND RIGHT IMAGE PAIR

The original image is processed by several different algorithms above, and the matching effect is shown in figure IX.

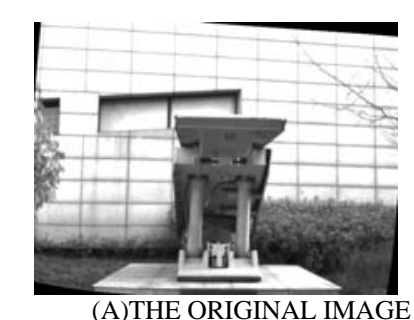

(A)THE ORIGINAL IMAGE

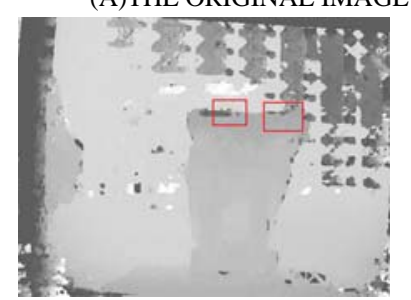

(C)ECA METHOD

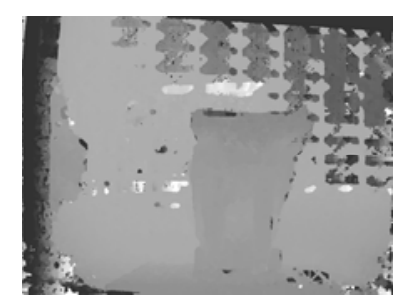

(B)ASW METHOD

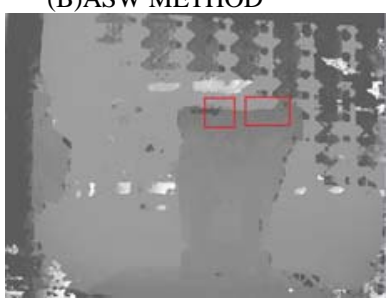

(D)REGIONAL ADAPTIVE METHOD
FIGURE IX. SIMULATION EXPERIMENT MATCHING EFFECT CHART

It can be seen from the red box of the effect map obtained through the simulation experiment that the regional adaptive stereo matching method significantly reduces the false matching in the edge area, the overall matching effect is improved, and the running time is not much different from the ECA algorithm. There is a certain gap between real-time positioning, but it is much faster than the ASW method.

\section{CONCLUSION}

Several stereo matching algorithms proposed in this paper can be applied to the study of the position of underground mine. Through experiments, it can be found that the improved algorithm has a good matching effect and the running time has been improved.

\section{ACKNOWLEDGMENT}

This project is supported by the National key research and development program (Grant No.2016YFC0801800).

\section{REFERENCES}

[1] Thrybom, L., et al., Future Challenges of Positioning in Underground Mines. IFAC-PapersOnLine, 2015. 48(10): p. 222-226.

[2] Wang, J., et al., Modeling of binocular stereo vision for remote coordinate measurement and fast calibration. Optics and Lasers in Engineering, 2014. 54: p. 269-274.

[3] Comlekciler, I.T., S. Gunes and C. Irgin, Artificial 3-D contactless measurement in orthognathic surgery with binocular stereo vision. Applied Soft Computing, 2016. 41: p. 505-514.

[4] Hamzah, R.A., et al., Improvement of stereo matching algorithm for 3D surface reconstruction. Signal Processing: Image Communication, 2018. 65: p. $165-172$.

[5] Hosni, A., M. Bleyer and M. Gelautz, Secrets of adaptive support weight techniques for local stereo matching. Computer Vision and Image Understanding, 2013. 117(6): p. 620-632.

[6] Gu, Z., et al., Local stereo matching with adaptive support-weight, rank transform and disparity calibration. Pattern Recognition Letters, 2008. 29(9): p. 1230-1235.

[7] De-Maeztu, L., A. Villanueva and R. Cabeza, Stereo matching using gradient similarity and locally adaptive support-weight. Pattern Recognition Letters, 2011. 32(13): p. 1643-1651.

[8] Nalpantidis, L. and A. Gasteratos, Biologically and psychophysically inspired adaptive support weights algorithm for stereo correspondence. Robotics and Autonomous Systems, 2010. 58(5): p. 457-464.

[9] Zhu, Z. and Q. Dai, Hybrid scheme for accurate stereo matching. Neurocomputing, 2017. 252: p. 24-33.

[10] $\mathrm{Hu}$ W, Zhang K, Sun L, et al. Virtual support window for adaptiveweight stereo matching[C]// Visual Communications and Image Processing. IEEE, 2011:1-4.

[11] Zhu, S., et al., Edge-preserving guided filtering based cost aggregation for stereo matching. Journal of Visual Communication and Image Representation, 2016. 39: p. 107-119.

[12] Tombari F, Mattoccia S, Stefano L D, et al. Near real-time stereo based on effective cost aggregation[C]// International Conference on Pattern Recognition. IEEE, 2009:1-4.

[13] Zhan Y, Gu Y, Huang K, et al. Accurate Image-Guided Stereo Matching With Efficient Matching Cost and Disparity Refinement[J]. IEEE Transactions on Circuits \& Systems for Video Technology, 2015, 26:1-1.

[14] Mozerov M G, Van d W J. Accurate Stereo Matching by Two-Step Energy Minimization[J]. IEEE Transactions on Image Processing A Publication of the IEEE Signal Processing Society, 2015, 24(3):1153.

[15] Hamzah, R.A., H. Ibrahim and A.H. Abu Hassan, Stereo matching algorithm based on per pixel difference adjustment, iterative guided filter and graph segmentation. Journal of Visual Communication and Image Representation, 2017. 42: p. 145-160.

[16] Li, L., et al., A MATLAB-based image processing algorithm for analyzing cupping profiles of two-layer laminated wood products. Measurement, 2014. 53: p. 234-239. 